\title{
Efficient cotransduction of tumors by multiple herpes simplex vectors: Implications for tumor vaccine production
}

\author{
Howard M. Karpoff, ${ }^{1}$ David Kooby, ${ }^{1}$ Michael D'Angelica, ${ }^{1}$ Jonathan Mack, ${ }^{1}$ David H. Presky, ${ }^{2}$ \\ Michael D. Brownlee, ${ }^{3}$ Howard Federoff, ${ }^{4}$ and Yuman Fong ${ }^{1}$ \\ ${ }^{1}$ Department of Surgery, Memorial Sloan-Kettering Cancer Center, New York, New York 10021; ${ }^{2}$ Department \\ of Inflammation/Autoimmune Diseases, Hoffmann-La Roche, Nutley, New Jersey 07110; ${ }^{3}$ Department of \\ Medicine, Albert Einstein College of Medicine, Bronx, New York 10461; and ${ }^{4}$ Department of Neurology, \\ Medicine, Microbiology, and Immunology, University of Rochester, Rochester, New York 14642.
}

\begin{abstract}
Many gene therapy strategies would be enhanced by efficient transfer of multiple genes into the same cell. Herpes simplex viral amplicon (HSV) vectors are good vehicles for gene transfer because they accommodate large pieces of foreign DNA and transfer genes rapidly and efficiently. The current studies examine whether efficient cotransduction of tumor cells can be accomplished using multiple HSV vectors in a manner useful for clinical gene therapy. Interleukin-12 (IL-12) exists as a heterodimer, with components (m35 and m40) coded for by genes on two separate chromosomes. We constructed HSV vectors carrying either IL12m35 (HSVm35) or IL12m40 (HSVm40) or both genes (HSVm75) separated by an internal ribosome entry site to assess whether gene transfer using a single HSV vector constructed to carry multiple genes has any advantage over gene transfer using multiple vectors that are each carrying single genes. Because IL-12 and IL-2 have been found to have synergistic antitumoral activity, we further analyzed the biologic activity of tumor cells cotransduced by separate HSV vectors carrying genes coding for these two cytokines. The results demonstrate that multiple genes can be inserted into the same cell efficiently using multiple HSV vectors, and that these vectors allow rapid production of tumor vaccines expressing multiple cytokine genes. Thus, gene transfer using HSV may not be limited by the size of the DNA that each vector can accommodate. Immunizations with tumors cotransduced with HSVm35 and HSVm40 were equally effective in eliciting a cytolytic T-lymphocyte response and in protecting against tumor growth in vivo as immunization with tumors treated with HSVm75. Immunization with tumors cotransduced with HSVm75 and HSVil2 was superior to immunization with tumors transduced with either alone. The combination of IL-2- and IL-12-secreting tumor cells may be used as an effective immunization strategy against solid tumors. Cancer Gene Therapy (2000) 7, 581-588
\end{abstract}

Key words: Tumor vaccine; interleukin; interleukin-2; interleukin-12; liver; herpes simplex virus.

$\mathrm{M}$ any gene therapy strategies depend upon transfer of multiple genes into the same cell. This will be particularly important for cancer treatment strategies involving the production of autologous tumor vaccines. A large body of data has demonstrated that experimental immunization using tumor cells engineered to secrete immunostimulatory cytokines such as interleukin-2 (IL$2)$, interferon- $\gamma$, or granulocyte-macrophage colonystimulating factor can provide lasting antitumoral immunity and protect against subsequent tumor challenge. ${ }^{1-5}$ Some studies have also shown such tumor vaccines to be effective against established macroscopic tumors. ${ }^{1,6,7}$ One current strategy attempts to further enhance the immunostimulatory properties of tumor vaccines by engineering tumor cells to produce multiple immunostimulatory cytokines or to produce combinations of

Received February 24, 1999; accepted July 25, 1999

Address correspondence and reprint requests to Dr. Yuman Fong, Memorial Sloan-Kettering Cancer Center, 1275 York Avenue, New York, NY 10021. E-mail address: FongY@MSKCC.org cytokines and leukocyte costimulatory proteins. It is hoped that local production of various immunostimulatory molecules in proximity to putative tumor antigens will activate heterogeneous tumor-suppressive mechanisms. ${ }^{5,8}$ Which combination of cytokines will prove synergistic in the immune therapy of cancers is under intense investigation. In addition, the most effective means of delivering multiple cytokines is currently unknown. Whether gene transfer using a single vector constructed to carry multiple genes has any major advantage over gene transfer using multiple vectors, each carrying a single gene, has yet to be determined.

IL-12 is an immunostimulatory cytokine that is particularly suitable for addressing these questions: it exists as a heterodimer, and its components (m35 and m40) are coded for by genes on two different chromosomes. ${ }^{9}$ In this study, we constructed herpes simplex virus amplicon (HSV) vectors carrying either IL12m35 (HSVm35) or IL12m40 (HSVm40) or both genes (HSVm75) separated by an internal ribosome entry site (IRES) (Fig 1) to assess the efficiency of gene transfer by a single vector 


\section{pHSV /IL12m75}

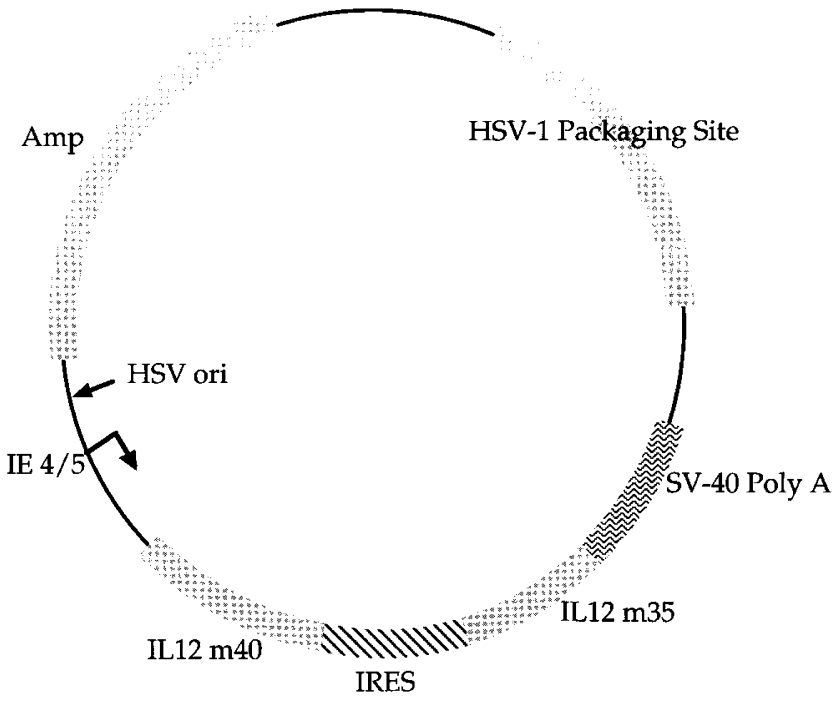

Figure 1. The HSVm75 plasmid. The plasmid was constructed by directionally cloning the m35 and m40 genes into HSV/PRPuc linked by an IRES.

carrying two genes compared with two vectors carrying the genes separately. We also examined the antitumoral efficacy of these constructs in vivo. IL-12 is a promising agent in enhancing T-cell activation, and IL-12 and IL-2 may have synergistic antitumoral activity. ${ }^{10-12}$ The current experiments examined the efficacy of immunization with tumor cells cotransduced by HSV vectors containing the genes for IL-12 and IL-2. The results demonstrate that cotransduced tumor cells produce high levels of these cytokines, and protect against liver tumor growth in a well-established model of hepatocellular carcinoma.

\section{MATERIALS AND METHODS}

\section{Animals}

Animals received care under approved protocols in compliance with Memorial Sloan-Kettering Cancer Center's Institutional Animal Care and Use Committee guidelines. Male Buffalo rats (National Cancer Institute, Bethesda, Md) were housed in a temperature- $\left(\mathrm{T}=22^{\circ} \mathrm{C}\right)$ and humidity-controlled room with a 12-hour day/night cycle and were provided food (P.M.I. Mills, St. Louis, Mo) and water ad libitum. Food consumption and body weight were measured.

\section{Tumor}

The Morris hepatoma McA-RH7777 (American Type Culture Collection, Manassas, Va) was used in these experiments. This syngeneic tumor was maintained in culture and periodically implanted in flanks to ensure tumorigenicity.

\section{Splenocyte isolation}

Splenectomy was performed under sterile conditions. Each spleen was placed in a Petri dish with $10 \mathrm{~mL}$ of media (RPMI
1640 plus $10 \%$ fetal calf serum (FCS) plus $50 \mathrm{mg} / \mathrm{mL}$ gentamicin). Splenocytes were washed from the spleen by repeated injection with media, spun at $1500 \mathrm{rpm}$ for 5 minutes, and resuspended in $5 \mathrm{~mL}$ of red cell lysis solution $(8.8 \mathrm{~g} \mathrm{NaCl}, 50$ $\mathrm{mM}$ tris(hydroxymethyl)aminomethane, $10 \mathrm{~mL}$ Nonidet P-40, and $1.68 \mathrm{~g} \mathrm{NaF}$ per liter). After 1 minute, the solution was neutralized with $5 \mathrm{~mL}$ of media. Cells were spun at $1500 \mathrm{rpm}$ for 10 minutes, washed two times with media, and maintained in culture in media supplemented with $30 \mathrm{U} / \mathrm{mL}$ IL-2 (Chiron, Emeryville, Calif) for 2 days before use.

\section{Kupffer cell (KC) isolation}

KCs were obtained with standard collagenase perfusion and differential ultracentrifugation with an isoosmotic Percoll (Pharmacia Biotech, Uppsala, Sweden) gradient as described previously. ${ }^{13-15}$ The pellet containing KCs was resuspended in media plus $1 \%$ penicillin/streptomycin. Cells were counted, plated in 96-well plates (Costar, Cambridge, Mass), and incubated for 4 hours. Nonadherent cells were washed off with media. This procedure yields cells that adhere, take up India ink uniformly, and stain positive with Wright-Giemsa stain (Baxter, McGaw Park, Ill), confirming that this procedure produced KCs.

\section{HSV vectors}

The murine $I L 12 m 35$, murine $I L 12 m 40$, human $I L-2$, and $\mathrm{LacZ}$ genes were cloned directionally into HSV/PRPuc and packaged as described previously. ${ }^{16-18}$ To produce HSVm75, the $\mathrm{m} 35$ and $\mathrm{m} 40$ genes were cloned directionally into HSV/ PRPuc separated by an IRES fragment using appropriate restriction enzymes (Fig 1). HSV/PRPuc contains the HSV immediate early $4 / 5$ promoter, a multiple cloning site, and an simian virus 40 A sequence, and has been described previously. ${ }^{19-23}$ The RR1 cells used for the packaging of HSV amplicons were maintained in Dulbecco's modified Eagle's medium containing high glucose $(4.5 \mathrm{~g} / \mathrm{L}), 10 \%$ FCS, $1 \%$ penicillin/ streptomycin, and $400 \mathrm{fg} / \mathrm{mL}$ bioactive geneticin (G418; Life Technologies, Gaithersburg, $\mathrm{Md}$ ) at $37^{\circ} \mathrm{C}, 5 \% \mathrm{CO}_{2}$. RR1 cells are baby hamster kidney cells that have been stably transfected with the HSV IE3 gene; they were obtained from Dr. Paul Johnson (University of California San Diego, San Diego, Calif). ${ }^{22}$ D30EBA helper virus was prepared by growth on RR1 cells. D30EBA is a strain 17-derived IE3 mutant deleted from codons 83-1236; it was obtained from Dr. Roger Everett (MRC Virology Unit, Institute of Virology, Glasgow, UK). ${ }^{23}$ To package amplicon vectors, $3 \times 10^{6} \mathrm{RR} 1$ cells were plated in media containing 10\% FCS; after 4 hours, the cells were transfected by adding $40 \mu \mathrm{l}$ of Lipofectin (Life Technologies), waiting 5 minutes, and then adding the amplicon DNA solution dropwise (30 $\mu \mathrm{g}$ at $1 \mu \mathrm{g} / \mu \mathrm{l}$ in Dulbecco's modified Eagle's medium). After 6 hours, plates were fed with media containing $5 \%$ FCS. At $\sim 20$ hours posttransfection, 50-100 $\mu \mathrm{l}$ of D30EBA virus was added to achieve a multiplicity of infection (MOI) of 0.2 . A total of $5 \mathrm{~mL}$ of complete media with $5 \%$ FCS was added to each plate after 1 hour. Amplicon virus stocks were harvested 2 days later. After overnight storage at $-70^{\circ} \mathrm{C}$, fresh RR1 cells $\left(4 \times 10^{6}\right.$ cells per 60 -mm plate) were infected with sonicated and warmed $\left(34^{\circ} \mathrm{C}\right)$ virus stock. After 2 days, the stocks were harvested and stored for subsequent use. HSVlac virus stocks were titrated by an expression assay. In brief, NIH 3 T3 cells were plated $\left(2 \times 10^{5}\right.$ cells/well in a 24 -well plate $)$ and infected with increasing volumes of an HSV virus stock in duplicate. At 24 hours postinfection, cells were fixed and stained with the chromogenic substrate 5-bromo-4-chloro-3indolyl $\beta$-D-galactoside using standard methods. ${ }^{17}$ The number of 5-bromo-4-chloro-3-indolyl $\beta$-D-galactoside-positive 
(blue) cells were counted. Titers are expressed as the number of blue-forming units per milliliter. The D30EBA helper virus in each stock was titrated by plaque assay on RR1 cells. HSVm35, HSVm40, HSVm75, and HSVil2 were titrated by a slot blot assay as described previously. ${ }^{24}$ For slot blot analysis, viral DNA was extracted from packaged virus by phenol/ chloroform twice, ethanol-precipitated with single-strand calf thymus DNA as carrier, denatured at room temperature with $0.2 \mathrm{~N} \mathrm{NaOH}$ and $0.5 \mathrm{M} \mathrm{NaCl}$ for 10 minutes, and loaded onto a nylon membrane with a slot blot apparatus. The membrane was then baked for 2 hours at $65^{\circ} \mathrm{C}$ and probed with a ${ }^{32}$ P-labeled 435-bp SspI and PvuI fragment containing part of the $\beta$-lactamase gene from pBR322 (nucleotides 3733-4168). After stringent washing $(0.1 \times$ standard saline citrate twice for 15 minutes), blots were exposed to x-ray film; next, various timed exposures were taken and densitometrically scanned (LKB Ultroscan, Uppsala, Sweden). Band densities between HSVlac and other viral stocks were compared; in addition, the titer of HSVm35, HSVm40, HSVm75, or HSVil2 was calculated from the density relative to HSVlac, given that this latter amplicon was titrated by an expression assay (blue-forming units on NIH 3 T3 cells). The titers of viral stocks are expressed as particles per milliliter. Titers of amplicon stocks were between 0.8 and $2 \times 10^{6}$ particles/mL. D30EBA titers in stocks ranged from $5 \times 10^{6}$ to $6 \times 10^{7}$ plaque-forming units $/ \mathrm{mL}$. Recombination for wild-type revertants was monitored by plaque assay on Vero cells and occurred at a frequency of $1 \times 10^{-6}$.

Efficiency of transduction with HSVm35 + HSVm40 vs. HSVm75: In vitro production of cytokines

To assess the in vitro production of cytokines, $10^{6}$ hepatoma cells per $2 \mathrm{~mL}$ were plated in 6-well plates (Costar), radiated with 10,000 rad, and rested for 1 hour. Cells were then exposed to HSVm35, HSVm40, HSVm35 plus HSVm40, HSVm75, HSVlac, or media for 20 minutes at an MOI of between 1 and 4 and subsequently washed two times with media. Cell culture supernatants were harvested on days $1,2,4,5$, and 7 after exposure, and cytokine levels were measured by an enzymelinked immunosorbent assay that was specific for the heterodimeric IL-12 protein. ${ }^{25}$ All conditions at each timepoint were tested at least in quadruplicate.

To prove that cells producing either $\mathrm{m} 35$ or $\mathrm{m} 40$ cannot be admixed to produce mature IL $12 \mathrm{~m} 75$ protein, the following experiment was performed. Tumor cells were transduced with either HSVm35 or HSVm40 at an MOI of between 1 and 4 for 20 minutes. These cells were then washed and combined. Cell culture supernatants were harvested on days $1,2,4,5$, and 7 after exposure, and cytokine levels were measured.

\section{Effect of vaccination on hepatic tumor growth}

Hepatoma cells in culture were radiated with 10,000 rad, rested for 1 hour, and subsequently exposed to HSVil2, HSVm75, HSVil2 plus HSVm75, HSVm 35 plus HSVm40, or media for 20 minutes at an MOI of 1-4. Previous studies in our laboratory have shown no significant effect of vaccination with radiated cells or vaccination with radiated cells transduced by HSVlac compared with injection of media alone. ${ }^{4}$ Cells were washed two times with media, and $10^{6}$ cells $/ 200 \mu \mathrm{l}$ were injected intrasplenically. Previous experiments have demonstrated that intrasplenic vaccination is more effective than either intraperitoneal or subcutaneous vaccination. ${ }^{26}$ An additional group received two populations of cells: A total of $10^{6}$ HSVil2-transduced cells and $10^{6} \mathrm{HSVm} 75$-transduced cells. A total of 10 animals were included in each treatment group. On day 21 , all animals received a challenge of $5 \times 10^{5}$ hepatoma cells/200 $\mu \mathrm{l}$ intraportally. This model produces uniform numbers of tumors within the liver that can be counted on day 20 after tumor challenge., ${ }^{45}$ Operative procedures were performed under pentobarbital anesthesia $(25 \mathrm{mg} / \mathrm{kg}$ intraperitoneally) via midline abdominal incision. Animals were sacrificed at 20 days after portal tumor challenge, and tumor nodules were counted.

\section{Effect of vaccination on splenocyte and KC function}

Four animals in each treatment group underwent vaccination as described above, and splenocytes and KCs were harvested on day 21 postvaccination and assessed for tumoricidal activity by a standard Europium release assay. ${ }^{4,15}$ Briefly, tumoricidal activity was assessed by mixing effectors with Europiumlabeled tumor cells in vitro. Labeled cells were plated at a concentration of $5 \times 10^{4}$ cells $/ 100 \mu \mathrm{l} /$ well in a 96-well, Ubottom plate (Costar) containing effector cells in wells at varying effector to target (E:T) ratios. The plate was spun at $200 \mathrm{rpm}$ for 5 minutes, incubated for 4 hours at $37^{\circ} \mathrm{C}$, and spun at $500 \mathrm{rpm}$ for 5 minutes. A total of $20 \mathrm{~mL}$ of supernatant was transferred to a 96-well flat-bottom plate (Costar) already containing $180 \mu \mathrm{l} /$ well of Delfia enhancement solution (Wallac Oy, Turku, Finland). The plate was read in a 1232 Delfia fluorometer (Wallac Oy). Maximum lysis was measured by lysing cells with $1 \%$ Triton X-100. The percentage of specific lysis was calculated using the following formula: ([experimental release - spontaneous release]/[maximum release - spontaneous release ]) $\times 100$. Spontaneous release varied between $5 \%$ and $15 \%$ of the maximum release. Assays were performed in triplicate.

\section{Statistical analysis}

The statistical significance of differences in the number of liver nodules, as well as the percentage of percent specific lysis, expressed as mean \pm SEM, were analyzed using analysis of variance or Student's $t$ test (SPSS, Chicago, Ill) as appropriate.

\section{RESULTS}

Efficiency of transduction with HSVm35 and HSVm4O versus HSVm75: In vitro production of cytokines

Tumor cells transduced with the combination of HSVm 35 and HSVm 40 were compared with those transduced with HSVm75 to determine the efficiency of cotransduction by two separate vectors. Control cells exposed to HSV not containing any cytokine genes do not produce IL-12. Furthermore, IL-12 production was not detected in cells transduced with either HSVm35 or HSVm40 alone. Furthermore, cells transduced with HSVm35, when admixed with cells transduced with HSVm40, did not produce IL-12 protein recognizable by the ELISA for the heterodimeric protein. Transduction of tumor cells by HSVm75 was efficient and led to the production of high levels of IL-12 by the transduced tumor cells. An 20-minute exposure to $\mathrm{HSVm} 75$ resulted in production of $5000 \pm 400 \mathrm{pg} / 10^{6}$ cells/24 hours. Transduction using two vectors (HSVm35 and HSVm40) produced levels of IL-12 similar to transduction using a single vector carrying both genes (HSVm75) (Fig 2). IL-12 levels peaked on day 1 and decreased 


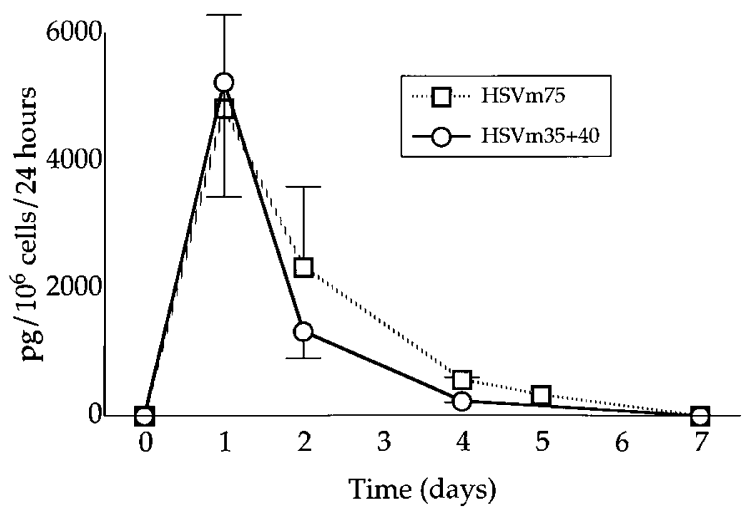

Figure 2. In vitro production of IL-12. A total of $10^{6}$ radiated Morris hepatoma cells were exposed to HSVm75 or HSVm35 plus HSVm40 for 20 minutes. Supernatants were evaluated for cytokine production by ELISA. When cells exposed separately to HSVm35 or HSVm40 were washed and then combined, no mature IL-12 protein was produced.

thereafter. Thus, the high efficiency of transduction of HSV vectors allows for cotransduction of cells using separate HSV vectors when expression of multiple foreign genes within the same cell is desired.

\section{Effect of vaccination by cytokine-producing tumors on hepatic tumor growth}

To evaluate the efficacy of tumor cells transduced with multiple HSV vectors in eliciting an effective antitumoral response, tumors transduced with various combinations of $\mathrm{HSV}$ vectors were tested in a standard animal model $^{4,15}$ of liver tumor metastases. In the control animals treated by immunization with tumor cells transduced by HSV vectors carrying no cytokine genes, $\sim 100$ tumor nodules were noted in the liver at 3 weeks after an intraportal challenge with $10^{6}$ hepatoma cells (Fig 3). Animals immunized with cells transduced by the combination of HSVm35 plus HSVm40 or by HSVm75 had significantly fewer tumor nodules than control animals (Fig 3, $P<.001$ ). Immunization with tumor cells cotransduced with HSVm35 and HSVm40 was as effective as treatment with tumors transduced by HSVm75.

In the same model, comparisons were also made with regard to tumor growth after vaccination with tumor cells transduced by HSVil2, by HSVm75, or by the combination to determine whether IL-2 and IL-12 expression produces synergistic effects on tumor suppression. Confirming previous studies, vaccination with tumor cells transduced with HSVil2 significantly reduced subsequent liver tumor growth after portal tumor challenge. Vaccination with two tumor cell populations, one secreting IL-2 and one secreting IL-12, was more effective than vaccination with a single population of cytokine-secreting cells. Most importantly, vaccination with a single population of cells transduced by both HSVil2 and HSVm75 was the most effective treatment; this treatment was much more effective than vaccination with the

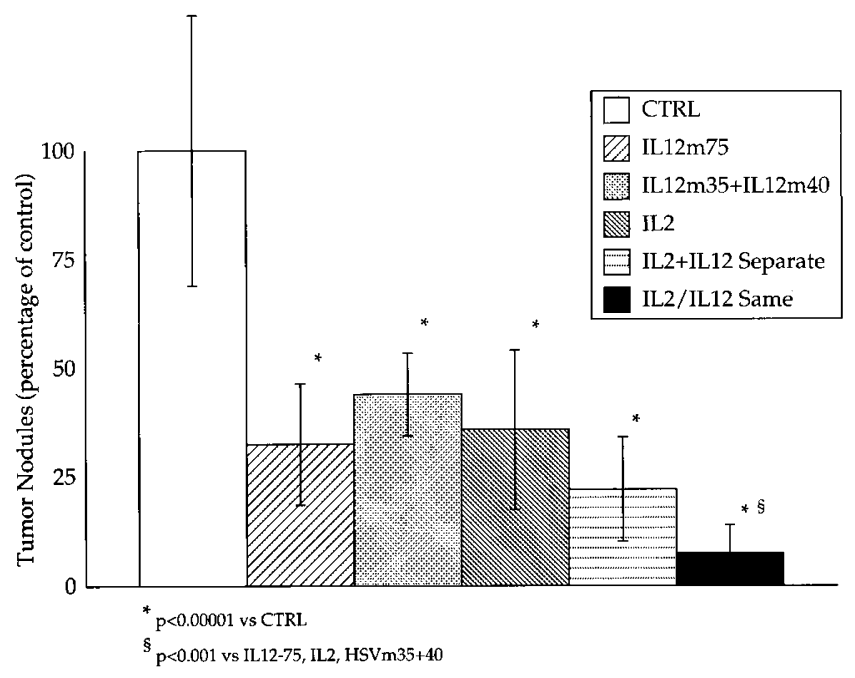

Figure 3. Number of hepatic tumor nodules at 3 weeks after splenic injection of hepatoma cells. Animals underwent vaccination with radiated cells transduced by media (control (CTRL)), HSVm75, HSVm35 plus HSVm40, HSVil2, or HSVm75 plus HSVil2. On day 21, all animals were challenged with replicating tumors. Animals were sacrificed at 20 days postchallenge, and tumor nodules within the liver were counted. $*, P<.0001$ versus CTRL; $\S, P<.01$ versus IL12m75, IL-2, and HSVm35 plus HSVm40; $n=10$ per group.

combination of two separate tumor vaccines each transduced by HSVil2 or HSVm75 $(P<.001$, Fig 3$)$.

\section{Effect of vaccination on splenocyte and $K C$ function}

Splenocytes and KCs were isolated from an animal vaccinated with tumor cells transduced with the various HSV vectors to determine the relative efficacy of the tumor cells in generating a cellular immune response to tumor. Splenocytes from animals vaccinated by either HSVil2 or HSVm75 had significantly greater killing of targets than splenocytes from animals vaccinated by radiated cells $(P<.01$, Fig 4A). Splenocytes from

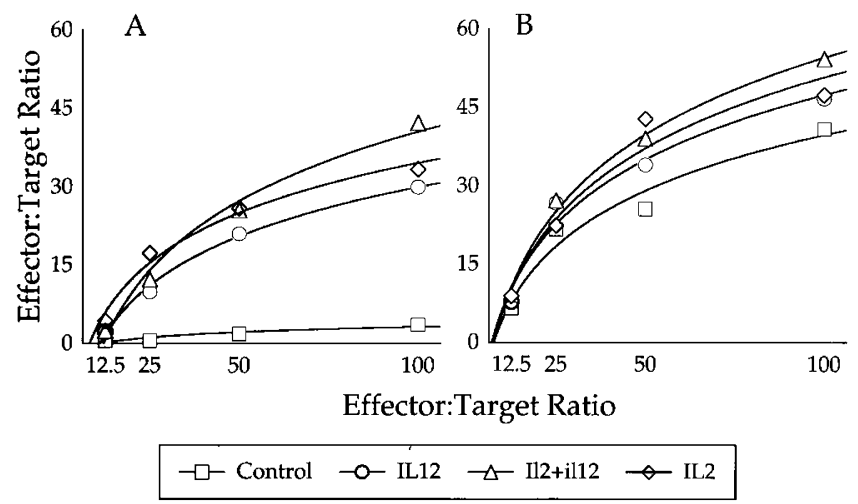

Figure 4. Lysis of Morris hepatoma cells by splenocytes (A) and KCs (B) harvested from animals vaccinated with radiated cells transduced by HSVil2, HSVm75, HSVil2 plus HSVm75, or media (Control). A: Splenocytes harvested from animals vaccinated with cytokine-secreting cells had significantly increased cytolytic activity $(P<.05)$ compared with control. B: KCs harvested from animals vaccinated with cytokine-secreting cells had significantly increased cytolytic activity $(P<.05)$ compared with control. 
animals vaccinated by cells cotransduced by $\mathrm{HSVm} 75$ and HSVil2 had significantly greater killing of targets than splenocytes from animals vaccinated by a single cytokine $(P<.01$ Fig $4 \mathrm{~A})$ at an $\mathrm{E}: \mathrm{T}$ ratio of 100:1.

KCs from rats vaccinated with HSVil2 or HSVm75 had significantly greater tumoricidal activity than KCs from controls $(P<.05)$ at an E:T ratio of 50:1. KCs from animals vaccinated by cells transduced by HSVm75 and HSVil2 had significantly greater killing of targets than KCs from animals vaccinated by a single cytokine at an E:T ratio of 100:1 $(P<$ .05 , Fig 4B).

\section{DISCUSSION}

Many successful immunotherapy strategies have used tumor cells engineered to secrete cytokines locally. This is done to reduce the side effects associated with the systemic administration of cytokines, ${ }^{27}$ and with the hope that local production of cytokines in the area of putative tumor antigens may generate a more vigorous tumor-specific immune response ${ }^{28,29}$ than would be achievable by systemic cytokine administration. In many experimental models, such cytokine-secreting tumor cells exhibited decreased tumorigenicity. ${ }^{1,2,30}$ Immunization with irradiated tumor cells secreting a single cytokine has also been shown to be effective in preventing the growth of subsequently administered tumor cells, $, 1,2,6$ even producing regression of established tumors in some cases. $6,7,30-33$ In an attempt to further augment the immunostimulatory activity of the tumor vaccines, one direction of current research seeks to determine whether immunization using tumors secreting multiple cytokines or tumors secreting combinations of cytokines and other immunostimulatory proteins, such as chemokines or cell adhesion molecules, may be a more effective treatment. Efficient and clinically relevant gene transfer strategies for the transfer of multiple genes would not only facilitate such research but may also have human therapeutic application. The HSV vectors are attractive candidates as vehicles for gene transfer in the production of autologous tumor vaccines because of their high efficiency and rapidity for gene transfer to a wide variety of cell types. ${ }^{4,26}$ The current study also demonstrates HSV vectors to be promising vehicles for multiple gene transfer in tumor vaccine production. The ease of administration of a single HSV vector containing several coding sequences linked together by IRES fragments was compared with administration of multiple vectors carrying the various genes separately. The results demonstrate that a 20-minute exposure of tumor cells to multiple HSV vectors, with each carrying separate genes, results in high-efficiency production of tumor vaccines that not only secrete multiple cytokines at biologically relevant levels but also produce in vivo antitumoral effects. These findings have important practical implications. Gene transfer using multiple HSV vectors carrying distinct candidate therapeutic genes allows ease of testing and ease of clinical application of combination gene therapeutic approaches.

The levels of IL-12 production by the HSV-trans- duced cells in this study are similar to or higher than those obtained by retroviral transduction. ${ }^{34,35}$ Using a retroviral vector, Tahara et $\mathrm{al}^{36}$ showed IL-12 production to be $4400 \mathrm{pg} / 10^{6}$ cells $/ 24$ hours in $3 \mathrm{~T} 3$ cells. In studies exposing dendritic cells to retroviral vectors for 6 hours on 3 consecutive days, production was $\sim 80 \mathrm{pg} / \mathrm{mL} /$ $10^{6}$ cells/24 hours. ${ }^{37}$ Most of these studies selected out only those cells that were successfully transduced by the retroviral vectors before assaying for IL-12 production. Such a process of retrovirally mediated gene transfer and cell selection is more cumbersome than the 20minute exposure to HSV used in the current studies. Recent publications using adenovirally mediated gene transfer reported higher levels of IL-12 production, but much higher MOIs were used. Chen et $\mathrm{al}^{38}$ used an adenoviral vector with an MOI of 500 to achieve $400 \mathrm{ng}$ of production. Bramson et $\mathrm{al}^{39}$ elicited the production of $\sim 300 \mathrm{ng} / 10^{6}$ cells/24 hours, but an MOI of 30 was used in these experiments. Hemmi et $\mathrm{al}^{40}$ used different cell lines and MOIs of up to 5000. One cell line, M950504, had production of $1 \mathrm{ng} / 10^{6}$ cells/24 hours at an MOI of 100. At the MOI of 1 used in the current studies, $\sim 4$ $\mathrm{ng} / 10^{6}$ cells $/ 24$ hours of IL-12 was produced. Furthermore, this level of production produced a significant biologic antitumoral effect.

IL-12, originally identified as natural killer (NK) cell-stimulating factor, ${ }^{9}$ is a cytokine that is particularly suited to a study of multiple gene transfer because of its heterodimeric structure. This immunostimulatory cytokine is coded by two genes that reside on separate chromosomes, and simultaneous cellular expression of both is necessary for the production of biologically functional protein. In the current study, transduction with HSVm 35 plus HSVm 40 produced roughly the same amount of IL-12 protein as transduction with HSVm75, demonstrating the high-efficiency cotransduction of tumors by two separate HSV vectors. Such cotransduction resulted in production of IL-12 that was biologically active in vitro and in vivo. Cotransduction of cells with multiple HSV vectors can be used to produce highefficiency transfer of multiple genes when simultaneous expression of multiple foreign genes is desired. On a practical level, such simultaneous cotransduction can enhance gene transfer strategies by reducing the need for complicated constructs when multiple gene transfer is desired. In addition, such cotransductions may increase the limits with regard to the size of genetic material that may be transferred by HSV, because the size of the genetic material transferred is no longer limited by the capacity of a single HSV vector.

IL-12 is also well suited for the current studies because of its novel immunostimulatory properties and its potential utility as an anti-cancer therapeutic agent. IL-12 has been shown to enhance the cytolytic activity of a number of cell types, including T cells, NK cells, and macrophages. ${ }^{9}$ Systemic administration of this immunostimulatory cytokine has been shown to result in antitumoral activity in animal models. ${ }^{8,12,41}$ Investigators have also tried to elicit high local production of IL-12 by infusing cultured fibroblasts genetically engineered to 
uniformly produce IL-12 along with irradiated tumor cells. ${ }^{10,42}$ This latter approach has also demonstrated antitumoral efficacy in animal models. ${ }^{41}$ The current study indicates that IL-12 production at local tumor sites can be achieved by direct HSV-mediated gene transfer. Production of tumor vaccines by transducing irradiated cells using HSV results in a high production of IL-12 that results in a systemic anti-cancer response. The ease with which such IL-12-secreting tumor vaccines can be produced also encourages studies of this approach for autologous tumor vaccine production in humans.

The combination of IL-2 and IL-12 has been shown to be synergistic in its antitumoral effects. ${ }^{10-12}$ The two cytokines use parallel and complementary signaling pathways. For example, IL-2 and IL-12 may have distinct signaling pathways leading to the induction of interferon- $\gamma$ and granulocyte-macrophage colony-stimulating factor expression. ${ }^{43}$ Human peripheral blood mononuclear cells treated with IL-2 and IL-12 kill neuroblastoma target cells in vitro more effectively than either alone. ${ }^{4}$ IL-2 and IL-12 synergize to stimulate NK activity in lymphocytes, ${ }^{45}$ and to more efficiently generate tumor-specific cytolytic T lymphocytes. ${ }^{46}$ In studies of the cytolytic responses of peripheral blood mononuclear cells, Rashleigh et $\mathrm{al}^{47}$ showed that the combination of IL-2 and IL-12 consistently produced greater activation of cytolysis than either cytokine alone. Combination therapy with vaccination using irradiated glioma cells engineered to produce IL-2 and systemic recombinant IL-12 has proved to be more effective than recombinant IL-12 treatment alone. ${ }^{48}$ Experiments in a murine renal cell carcinoma model showed that the systemic administration of IL-2 and IL-12 had greater antitumoral efficacy than either IL-2 or IL-12 alone. ${ }^{49}$ We took advantage of the ability of HSV vectors to cotransduce cells with high efficiency to evaluate the effects of tumor vaccines secreting the combination of IL-2 and IL-12 on hepatic tumor growth and to further validate the practicality of multiple gene delivery. In the current study, the use of an IL12m75- and IL-2-secreting cytokine vaccine was more effective than vaccination with cells secreting either of the cytokines. Vaccination with cytokine-producing tumor cells was associated with a measurable immune response. There was a lymphocyte immune response, as evidenced by the greater tumoricidal activity of splenocytes from vaccinated animals. In addition, there was a moderate effect of vaccination on the modulating $\mathrm{KC}$ response, consistent with the profile of these tissue-based macrophages. ${ }^{15,50}$ Such vaccinations also decreased the establishment and growth of the tumor in the liver after subsequent challenge with highly tumorigenic doses of unmodified tumor cells. Vaccination of cotransduced cells was also more effective than introducing two separate populations of single cytokinesecreting cells.

Our prior studies have demonstrated the ease of extracting tumor cells, transducing them with HSV vectors, and returning the transduced cells as a tumor vaccine to the host. ${ }^{26,51}$ HSV vectors are particularly good for this clinical application, because they can transduce irradiated, nonreplicating cells rapidly and efficiently. The current study demonstrates a practical and feasible way of creating vaccines secreting multiple products. The generation of tumor vaccines engineered to secrete multiple cytokines using multiple HSV vectors is simple and effective. It avoids the difficulties inherent in creating complex gene constructs, and it allows the construction of customized vaccines using multiple vectors from a library of single or dual gene-containing vectors. A process of producing autologous tumor vaccines that involves a 20-minute transduction of irradiated tumor is clinically practicable. These data encourage future studies of combinations of cytokines in directed vaccine gene therapy in the adjuvant treatment of patients with various cancers.

\section{ACKNOWLEDGMENTS}

We thank Dr. Ueli Gubler of Hoffmann-La Roche for providing the m35 and m40 genes and Yong-Jia You, MS, for his technical assistance in these projects. This work was supported in part by Grants RO1HD31300 (to H.F.), RO1CA76416 (to Y.F.), RO1CA72632 (to Y.F.), and RO1CA61524 (to Y.F.) from the National Institutes of Health, and by the Sara Chait Foundation (to Y.F.).

\section{REFERENCES}

1. Dranoff G, Jaffee E, Lazenby A, et al. Vaccination with irradiated tumor cells engineered to secrete murine granulocyte-macrophage colony-stimulating factor stimulates potent, specific, and long-lasting anti-tumor immunity. Proc Natl Acad Sci USA. 1993;90:3539-3543.

2. Gansbacher B, Zier K, Daniels B, Cronin K, Bannerji R, Gilboa E. Interleukin-2 gene transfer into tumor cells abrogates tumorigenicity and induces protective immunity. J Exp Med. 1990;172:1217-1224.

3. Hock H, Dorsch M, Kunzendorf U, et al. Vaccinations with tumor cells genetically engineered to produce different cytokines: effectivity not superior to a classical adjuvant. Cancer Res. 1993;53:714-716.

4. Karpoff HM, D'Angelica M, Blair S, Brownlee MD, Federoff H, Fong Y. Prevention of hepatic tumor metastases in rats with herpes viral vaccines and $\gamma$-interferon. J Clin Invest. 1997;99:799-804.

5. Pardoll DM. Genetically engineered tumor vaccines. Ann N Y Acad Sci. 1993;690:301-310.

6. Porgador A, Tzehoval E, Vadai E, Feldman M, Eisenbach L. Immunotherapy via gene therapy: comparison of the effects of tumor cells transduced with the interleukin-2, interleukin-6, or interferon- $\lambda$ genes. J Immunother. 1993; 14:191-201.

7. Saito S, Bannerji R, Gansbacher B, et al. Immunotherapy of bladder cancer with cytokine gene-modified tumor vaccines. Cancer Res. 1994;54:3516-3520.

8. Rao JB, Chamberlain RS, Bronte V, et al. IL-12 is an effective adjuvant to recombinant vaccinia virus-based tumor vaccines: enhancement by simultaneous B7-1 expression. J Immunol. 1996;156:3357-3365.

9. Brunda MJ. Interleukin-12. J Leukoc Biol. 1994;55:280288.

10. Pappo I, Tahara H, Robbins PD, et al. Administration of 
systemic or local interleukin-2 enhances the anti-tumor effects of interleukin-12 gene therapy. J Surg Res. 1995;58: 218-226.

11. Chang L, Bruner V, van de Ven C, Cairo MS. Synergistic enhancement of natural killer (NK) cell activity with cord blood by IL2 + IL12: a future immunotherapy approach post cord BMT. Proc Annu Meet Am Assoc Cancer Res. 1995;36:466. Abstract A2773.

12. Vagliani M, Rodolfo M, Cavallo F, et al. Interleukin 12 potentiates the curative effect of a vaccine based on interleukin-2-transduced tumor cells. Cancer Res. 1996;56: 467-470.

13. Kutteh WH, Rainey WE, Carr BR. Regulation of interleukin-6 production in human fetal Kupffer cells. Scand J Immunol. 1991;33:607-613.

14. West MA, Keller GA, Xerra FB, Simmons RL. Killed Escherichia coli stimulates macrophage-mediated alterations in hepatocellular function during in vitro coculture: a mechanism of altered liver function in sepsis. Infect Immun. 1985;49:563-569.

15. Karpoff HM, Tung C, Ng B, Fong Y. $\gamma$-interferon protects against hepatic tumor growth in rats by increasing Kupffer cell tumoricidal activity. Hepatology. 1996;24:374-379.

16. Geller AI, Keyomarsi K, Bryan J, Pardee AB. An efficient deletion mutant packaging system for defective herpes simplex virus vectors: potential applications to human gene therapy and neuronal physiology. Proc Natl Acad Sci USA. 1990;87:8950-8954.

17. Geller AI, Breakefield XO. A defective HSV-1 vector expresses Escherichia coli $\beta$-galactosidase in cultured peripheral neurons. Science. 1988;241:1667-1669.

18. Federoff JH. Growth of replication-defective herpes virus amplicon vectors and their use for gene transfer. In: Spector DL, Leinwand L, Goldman R, eds. Cell Biology: A Laboratory Manual. Plainview, NY: Cold Spring Harbor Laboratory Press; 1996.

19. Bergold P, Cassaccia-Bonnefil P, Xiu-Liu Z, Federoff H. Transsynaptic neuronal loss induced in hippocampal slice cultures by a herpes simplex virus vector expressing the GluR6 subunit of the kainate receptor. Proc Natl Acad Sci USA. 1993;90:6165-6169.

20. Linnik M, Zahos P, Geschwind M, Federoff H. Expression of bcl-2 from a defective herpes simplex virus-1 vector limits neuronal death in focal cerebral ischemia. Stroke. 1995;26:1670-1675.

21. Xu H, Federoff H, Maragos J, Parada LF, Kessler J. Viral transduction of trk $A$ into cultured nodose and spinal motor neurons conveys NGF responsiveness. Dev Biol. 1994;163:152-161.

22. Johnson P, Miyanohara A, Levine F, Cahill T, Friedmann T. Cytotoxicity of a replication-defective mutant of herpes simplex virus type I. J Virol. 1992;66:2952-2965.

23. Paterson T, Everett RA. A prominent serine-rich region in Vmw175, the major transcriptional regulator protein of herpes simplex virus type 1, is not essential for virus growth in tissue culture. J Gen Virol. 1990;71:1775-1783.

24. Expression of neurotrophic genes from herpes simplex virus type I vectors modifying neuronal phenotype. In: Geschwind M, Lu B, Federoff HJ, Flanagan TR, Emerich DF, Winn SR, eds. Providing Pharmacological Access to the Brain: Alternative Approaches. New York: Academic Press; 1994:462-482.

25. Gately MK, Chizzonite R, Presky DH. Measurement of Human and Mouse Interleukin 12. New York: John Wiley \& Sons; 1995;1:6.16.1-6.16.15.

26. Tung C, Federoff HJ, Brownlee M, et al. Rapid production of interleukin-2-secreting tumor cells by herpes simplex virus-mediated gene transfer: implications for autologous vaccine production. Hum Gene Therapy. 1996;7:2217-2224.

27. Rosenberg SA. Adoptive immunotherapy for cancer. Sci Am. 1990;262:34-39.

28. Ley V, Langlade-Demoyen P, Kourilsky P, Larrson-Sciard E. Interleukin 2-dependent activation of tumor-specific cytotoxic T lymphocytes in vivo. Eur J Immunol. 1991;21: 851-854.

29. Rubin JT. Interleukin-2: its biology and clinical application in patients with cancer. Cancer Invest. 1993;11:460-472.

30. Porgador A, Tzehoval E, Katz A, et al. Interleukin 6 gene transfection into Lewis lung carcinoma tumor cells suppresses the malignant phenotype and confers immunotherapeutic competence against parental metastatic cells. Cancer Res. 1992;52:3679-3686.

31. Connor J, Bannerji R, Saito S, Heston W, Fair W, Gilboa E. Regression of bladder tumors in mice treated with interleukin-2 gene-modified tumor cells. J Exp Med. 1993; 177:1127-1134.

32. Vieweg J, Rosenthal FM, Bannerji R, et al. Immunotherapy of prostate cancer in the Dunning rat model: use of cytokine gene-modified tumor vaccines. Cancer Res. 1994; 54:1760-1765.

33. Golumbek PT, Lazenby AJ, Levitsky HI, et al. Treatment of established renal cancer by tumor cells engineered to secrete interleukin-4. Science. 1991;254:713-716.

34. Myers JN, Mank-Seymour A, Zitvogel L, et al. Interleukin-12 gene therapy prevents establishment of SCC VII squamous cell carcinomas, inhibits tumor growth, and elicits long-term antitumor immunity in syngeneic $\mathrm{C} 3 \mathrm{H}$ mice. Laryngoscope. 1998;108:261-268. Abstract.

35. Matsumoto G, Sunamura M, Shimamura H, et al. Adjuvant immunotherapy using fibroblasts genetically engineered to secrete interleukin 12 prevents recurrence after surgical resection of established tumors in a murine adenocarcinoma model. Surgery. 1999;125:257-264. Abstract.

36. Tahara H, Zitvogel L, Storkus WJ, et al. Effective eradication of established murine tumors with IL-12 gene therapy using a polycistronic retroviral vector. J Immunol. 1995;154:6466-6474. Abstract.

37. Ahuja SS, Mummidi S, Malech HL, et al. Human dendritic cell (DC)-based anti-infective therapy: engineering DCs to secrete functional IFN- $\gamma$ and IL-12. J Immunol. 1998;161: 868-876. Abstract.

38. Chen L, Chen D, Block E, et al. Eradication of murine bladder carcinoma by intratumor injection of bicistronic adenoviral vector carrying cDNAs for the IL-12 heterodimer and its inhibition by the IL-12 p40 subunit homodimer. J Immunol. 1997;159:351-359. Abstract.

39. Bramson JL, Hitt M, Gallichan WS, et al. Construction of double recombinant adenovirus vector expressing a heterodimeric cytokine: in vitro and in vivo production of biologically active interleukin-12. Hum Gene Ther. 1996;7: 333-342. Abstract.

40. Hemmi S, Geertsen R, Mezzacassa A, et al. The presence of human Coxsackievirus and adenovirus receptor is associated with efficient adenovirus-mediated transgene expression in human melanoma cell cultures. Hum Gene Ther. 1998;9:2363-2373. Abstract.

41. Bruna MJ, Lusitro L, Warrier RR, et al. Antitumor and antimetastatic activity of interleukin-12 against murine tumors. J Exp Med. 1993;178:1223-1230.

42. Tahara H, Zeh HJ, Storkus WJ, et al. Fibroblasts genetically engineered to secrete interleukin 12 can suppress 
tumor growth and induce antitumor immunity to a murine melanoma in vivo. Cancer Res. 1994;54:182-189.

43. Ye J, Ortaldo JR, Conlon K, et al. Cellular and molecular mechanisms of IFN- $\gamma$ production induced by IL-2 and IL-12 in a human NK cell line. J Leukoc Biol. 1995;58:225233. Abstract.

44. Rossi AR, Pericle F, Rashleigh S, Janiec J, Djeu JY. Lysis of neuroblastoma cell lines by human natural killer cells activated by interleukin-2 and interleukin-12. Blood. 1994; 83:1323-1328.

45. DeBlacker-Hohe DF, Yamauchi A, Yu CR, et al. IL-12 synergizes with IL-2 to induce lymphokine-activated cytotoxicity and perforin and granzyme gene expression in fresh human NK cells. Cell Immunol. 1995;165:33-43. Abstract.

46. Mogi S, Ebata T, Setoguchi Y, et al. Efficient generation of autologous peripheral blood-derived cytotoxic T lymphocytes against poorly immunogenic human tumors using recombinant CD80-adenovirus together with interleukin 12 and interleukin 2. Clin Cancer Res. 1998;4:713-720.

47. Rashleigh SP, Kusher DI, Endicott JN, et al. Interleukins 2 and 12 activate natural killer cytolytic responses of peripheral blood mononuclear cells from patients with head and neck squamous cell carcinoma. Arch Otolaryngol Head Neck Surg. 1996;122:541-547. Abstract.

48. Kikuchi T, Joki T, Saitoh S, et al. Anti-tumor activity of interleukin-2-producing tumor cells and recombinant interleukin 12 against mouse glioma cells located in the central nervous system. Int J Cancer. 1999;80:425-430. Abstract.

49. Wigginton JM, Komschlies KL, Back TC, et al. Administration of interleukin 12 with pulse interleukin 2 and the rapid and complete eradication of murine renal carcinoma. J Natl Cancer Inst. 1996;88:38-43. Abstract.

50. Schuurman B, Heuff G, Beelan RH, Meyer S. Enhanced killing capacity of human Kupffer cells after activation with human granulocyte/macrophage-colony stimulating factor and interferon $\gamma$. Cancer Immunol Immunother. 1994;39: 179-184.

51. Raper SE, Grossman M, Rader DJ, et al. Safety and feasibility of liver-directed ex vivo gene therapy for homozygous familial hypercholesterolemia. Ann Surg. 1996; 223:116-126. 